\title{
Hentschel, Armin, und Peter Lohauß (2019): Wohnungsmärkte und Wohnungspolitik. Beiträge zur Kritik des Immobiliensektors
}

\author{
Marburg: Metropolis-Verlag. 342 Seiten. 29,80€
}

\section{Björn Egner}

Online publiziert: 14. April 2020

(C) Der/die Autor(en) 2020

Hentschel und Lohau $\beta$ haben einen spannenden Band vorgelegt, der sowohl für „Neueinsteiger“ in das Thema Wohnen einen guten Ausgangspunkt als auch für arrivierte Wohnungsforscher gut entwickelte Denkanstöße bietet. Die sechs behandelten Themen (sozialer Wohnungsbau, Mieterbewegungen, Wohnungspolitik als Wirtschaftssektor, Bodenpolitik, Wohnungsmärkte und der Einfluss der EU auf deutsche Wohnungspolitik) sind gut gegeneinander abgegrenzt und kommen weitgehend ohne thematische Dopplungen in der Ausführung aus. Dies ist nicht selbstverständlich angesichts eines Politikfeldes, das sich als relativ unübersichtlich darstellt und dabei sowohl starke Überlappungen mit vielen angrenzenden Politikfeldern bereithält als auch durch eine relativ träge Entwicklung gekennzeichnet ist. Sofort fällt beim Aufschlagen des Buches auf, dass die Umfänge der einzelnen Beiträge stark variieren. Dies hängt indes nicht nur mit der unterschiedlichen Tiefe zusammen, in der die einzelnen Aspekte besprochen werden, sondern auch mit der Komplexität und der politischen Reichweite der jeweiligen Themen.

So spannt denn auch der erste Beitrag zum sozialen Wohnungsbau einen großen historischen Bogen von der Zeit des Zweiten Weltkriegs über das Wirtschaftswunder und die Abschaffung der Wohnungsgemeinnützigkeit bis in die 2000er-Jahre und thematisiert die entscheidenden Weichenstellungen in Wirtschaft und Politik. Insbesondere der auf die Gegenwart bezogene Teil des Kapitels ist lesenswert, denn Hentschel gelingt es hier sehr gut, aktuelle Probleme der Wohnraumversorgung (Angebotslücken in Großstädten, starker Anstieg der Mietpreise etc.) mit der ursprünglichen Zielsetzung des Instruments zu vergleichen und auf die bestehenden Problemlagen hinzuweisen. Daraus leitet er jeweils handfeste politische Forderungen

B. Egner $(\triangle)$

Institut für Politikwissenschaft, Technische Universität Darmstadt, Darmstadt, Deutschland

E-Mail: bjoern.egner@tu-darmstadt.de 
ab, beispielsweise die Rücknahme der Schuldenbremsen, die er als „,fiskalpolitische Zwangsjacken“ (S. 84) bezeichnet.

Der zweite Beitrag beschäftigt sich mit einer noch relativ jungen gesellschaftlichen Entwicklung, nämlich dem Aufkommen der neuen Mieterbewegung. Vor dem Hintergrund der Frage, was Populismus ausmacht und ob es so etwas wie einen ,linken Populismus“ geben kann, widmet sich Hentschel dem Spannungsfeld zwischen traditioneller Vermittlung von Mieterinteressen durch den Deutschen Mieterbund und seines Berliner Landesverbands und neuen, ,,außerparlamentarisch“ und lokal organisierten Mieterbewegungen. Dabei wird deutlich, dass die Bündnisse zwischen traditionellen und neuen Organisationen nicht neu sind, aber auf unterschiedliche Einflusskanäle und -logiken ausgerichtet sind, die einen inneren Widerspruch in der Mieterbewegung erzeugen (S. 128-129). So richtet sich die traditionelle Mieterbewegung darauf, in einem quasikorporatistischen Arrangement mit Wohnungswirtschaft und Regierungen Kompromisse auszuhandeln, während die „neue“ Mieterbewegung statt Verhandelns stärker direkte Aktionen (Demonstrationen, Besetzungen, Gründung von Mietersyndikaten etc.) bevorzugt.

Im dritten Beitrag beschäftigt sich Hentschel mit der grundsätzlichen Frage, ob die gegenwärtigen Probleme auf den Wohnungsmärkten als Ausdruck von Staats- oder Marktversagen gelten können. Er kommt dabei zu dem Schluss, dass Wohnungspolitik historisch parallel etwa zur Rentenpolitik und zur Bildungspolitik betrachtet werden müsse, also Politikfeldern, in denen gesellschaftliche Gegenbewegungen den „Marktfundamentalismus“ (S. 168) der Neoliberalen zurückgedrängt haben.

Im vierten, umfangreichsten Aufsatz widmet sich Hentschel dem Kern des gegenwärtigen Problems auf den Wohnungsmärkten, nämlich der Steuer- und Bodenpolitik. Sehr gut nachvollziehbar seziert er hierfür die Anreizsysteme und Pathologien des aktuellen Steuer- und Bodenrechts und wie dieses zur „Finanzialisierung“ der Immobilien- und Wohnungsmärkte geführt hat. Zusätzlich thematisiert er noch die avisierte Grundsteuerreform, die - worauf er mit Recht genüsslich hinweist - durch die Rechtsprechung des Bundesverfassungsgerichts ausgelöst wurde und nicht durch eine politische Auseinandersetzung. Letztere sei aber - auch da liegt er m. E. richtig - die Bedingung dafür, dass die Grundsteuer gerechter werde, denn die Grundsteuer stellt die manifestierte Entscheidung in einem zentralen gesellschaftlichen Konflikt, den Bodenverhältnissen, dar. Hentschel bleibt allerdings nicht bei der deutschen Debatte stehen, sondern zeigt mit einem kurzen Exkurs nach Skandinavien (S. 260-263), wohin die Reise gehen kann, wenn Grundstücksspekulation und Renditeorientierung durch gemeinwohlverantwortliche Eckpfeiler in der Steuer- und Bodenpolitik eingehegt werden.

In den letzten beiden Beiträgen beschäftigen sich die Autoren mit dem Einfluss des europäischen und globalen Wirtschaftswachstums bzw. der Europäischen Union auf die deutsche Wohnungspolitik. Der einzige Beitrag von Lohauß im Band greift das Dreieck von allgemeinem Wirtschaftswachstum, Investitionstätigkeit und den damit verbundenen Renditeerwartungen und sozialer Polarisierung aus anderer Perspektive nochmals auf. Deutlich gezeichnet wird die aktuelle Situation in Deutschland insbesondere bei der pointierten Beschreibung der drei „Wellen“ der Finanzialisierung (S. 297-298), aber auch bei der vergleichenden Analyse der deutschen Wohnungsmärkte mit denen anderer ausgewählter EU-Mitgliedsstaaten. Im 
letzten Beitrag spannt Hentschel in einem kurzen Teil den Bogen über alle Teile und stellt die Fragen, ob nicht a) die Wohnungsknappheit inzwischen ein paneuropäisches Problem sei und b) die Europäische Union als Hüterin über den Binnenmarkt nicht längst die geeignete politische Arena für den Kampf um die Wohnungspolitik darstelle. Auf Basis des kurzen Exkurses kommt er jedoch schnell zu dem Schluss, dass Wohnungspolitik weiterhin eine nationale Verantwortung sei, da die Europäische Union genau hier den zentralen Mangel aufweise: Sie würde zwar einen Binnenmarkt herstellen und garantieren, die sozialstaatliche Dimension der Europäischen Union sei jedoch massiv unterentwickelt (S. 329-330).

Der Band ist insgesamt sehr zu empfehlen, da er sowohl vertiefende Analyse zu Einzelaspekten bietet als auch wieder zu den ,großen Fragen“ zurückkehrt, die das Politikfeld und die sozialwissenschaftliche Forschung zum Wohnen bis heute beschäftigen. Besonders wertvoll sind die Beiträge deshalb, weil sie klar und mit dem Verweis auf Empirie argumentieren und sich nicht scheuen, klare Empfehlungen auszusprechen.

Funding Open Access funding provided by Projekt DEAL.

Open Access Dieser Artikel wird unter der Creative Commons Namensnennung 4.0 International Lizenz veröffentlicht, welche die Nutzung, Vervielfältigung, Bearbeitung, Verbreitung und Wiedergabe in jeglichem Medium und Format erlaubt, sofern Sie den/die ursprünglichen Autor(en) und die Quelle ordnungsgemäß nennen, einen Link zur Creative Commons Lizenz beifügen und angeben, ob Änderungen vorgenommen wurden.

Die in diesem Artikel enthaltenen Bilder und sonstiges Drittmaterial unterliegen ebenfalls der genannten Creative Commons Lizenz, sofern sich aus der Abbildungslegende nichts anderes ergibt. Sofern das betreffende Material nicht unter der genannten Creative Commons Lizenz steht und die betreffende Handlung nicht nach gesetzlichen Vorschriften erlaubt ist, ist für die oben aufgeführten Weiterverwendungen des Materials die Einwilligung des jeweiligen Rechteinhabers einzuholen.

Weitere Details zur Lizenz entnehmen Sie bitte der Lizenzinformation auf http://creativecommons.org/ licenses/by/4.0/deed.de. 